\title{
Faire évoluer une revue scientifique dans un monde changeant
}

\author{
Bernard Pochet ${ }^{(1)}$, Gilbert Berben ${ }^{(2)}$, Cédric Vermeulen ${ }^{(3)}$ \\ (1) Université de Liège - Gembloux Agro-Bio Tech. CARE Uliège Library. Passage des Déportés, 2. BE-5030 Gembloux \\ (Belgique).E-mail : bernard.pochet@uliege.be \\ (2) Centre wallon de Recherches agronomiques. Département Valorisation des Productions. Unité Authentification et \\ Traçabilité. Bâtiment Henseval. Chaussée de Namur, 24. BE-5030 Gembloux (Belgique). \\ (3) Université de Liège - Gembloux Agro-Bio Tech. Ingénierie des biosystèmes (Biose). Laboratoire de Foresterie des \\ Régions tropicales et subtropicales. Passage des Déportés, 2. BE-5030 Gembloux (Belgique).
}

Cet article est distribué suivant les termes et les conditions de la licence CC-BY (http://creativecommons.org/licenses/by/4.0/ deed.fr)

Après un bref rappel historique, cet article présente la situation actuelle de la revue BASE, ses objectifs, ses enjeux, son fonctionnement et ses limites. BASE est une revue qui publie en Open Access des articles du domaine des sciences agronomiques au sens large et offre un service gratuit aux auteurs. Le comité de rédaction est particulièrement attentif à la transparence de ses processus de validation et d'édition. Les chiffres présentés permettent de situer BASE dans l'ensemble des revues scientifiques internationales. En fin d'article, après la description de quelques difficultés, plusieurs perspectives sont décrites. Elles devraient rencontrer les inconvénients du caractère multidisciplinaire de BASE.

Mots-clés. Revue académique, libre accès, facteur d'impact, publication scientifique, revue par les pairs.

\section{Developing a scientific journal in a changing world}

After a brief historical review, this article presents the current situation of the journal BASE, its objectives, its challenges, its functioning and its limitations. BASE is a journal that publishes articles in Open Access in the field of agronomic sciences in the broad sense and offers a free service to authors. The editorial board pays particular attention to the transparency of its validation and editing processes. The figures presented allow BASE to find its place alongside all international scientific journals. At the end of the article, after describing the difficulties encountered, several perspectives are described. These should address the possible disadvantages of the multidisciplinary nature of BASE.

Keywords. Academic journal, open access, impact factor, scholarly publishing, peer reviewing.

\section{INTRODUCTION}

À sa création, en 1997, dans son premier numéro, BASE et ses ambitions étaient présentés (Baudoin, 1997). En 2002, un éditorial faisait le point après cinq ans de publication (Pochet, 2002). En 2009, un autre éditorial annonçait l'entrée de BASE dans le Science Citation Index (Pochet \& Baudoin, 2009). Cette entrée a permis à BASE d'obtenir un Impact Factor et a été à l'origine d'une hausse significative de sa visibilité. Ce changement constitue depuis lors un facteur d'attrait auprès des auteurs. En effet, le nombre d'articles soumis annuellement est passé de 80, en 2007, à 279, cinq ans plus tard. Ce nombre est resté constant depuis.

Cet article a pour objet de refaire le point après deux décennies de publication, alors que la communication scientifique a énormément évolué. D'une part, la mise en cause, par une partie de la communauté scientifique, du facteur d'impact comme seul moyen d'évaluation de la qualité des publications (Satyanarayana, 2010 ; Casadevall \& Fang, 2014 ; Callaway, 2016) a mené à la création de métriques alternatives (Galligan \& Dyas-Correia, 2013). D'autre part, la mise en avant des faiblesses du peer review « classique » (Margalida $\&$ Colomer, 2016 ; Murphy, 2016) et les nombreuses expériences tentées vers des systèmes de validation ouverts (Bon, 2015) ont interpellé les scientifiques. Mais surtout, les avancées majeures de l'Open Access, avec l'avènement des revues qui, comme BASE, offrent un service gratuit aux auteurs et un accès totalement libre aux articles publiés, ont révolutionné un système jusque-là dominé par des éditeurs payants ayant acquis une situation de quasi-monopole. 
Ce mouvement s'accompagne malheureusement d'un cortège de problèmes comme l'apparition des éditeurs prédateurs (Butler, 2013) ou des APC (Article Processing Charges) avec des éditeurs qui demandent plusieurs milliers d'euros aux auteurs pour publier leurs articles (Crawford, 2017; Wenzler, 2017). Le nombre croissant de faux facteurs d'impact (voir: https:// predatoryjournals.com/metrics/) contribue également à compliquer la situation. Plus que jamais, les revues doivent faire preuve de transparence et démontrer la qualité de leurs processus de validation et d'édition des articles et l'éthique de leurs démarches. BASE s'inscrit totalement dans ce mouvement.

\section{OBJECTIFS}

BASE a succédé, en 1997, au « Bulletin des Recherches Agronomiques de Gembloux » et, dès le départ, s'est fixé comme objectif d'être une vitrine de la recherche au «Centre de Recherches agronomiques de Gembloux », devenu depuis "Centre wallon de Recherches agronomiques», et de la «Faculté des Sciences agronomiques de Gembloux », devenue

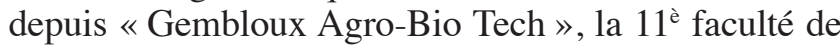
l'Université de Liège.

Ce rôle de vitrine fait de BASE une revue largement multidisciplinaire puisqu'elle publie des articles originaux dans les domaines des sciences agronomiques, des forêts, de la nature et des paysages, des sciences et des technologies de l'environnement, de la chimie et des bio-industries.

BASE est donc avant tout un service offert aux chercheurs de la communauté scientifique gembloutoise. BASE y est la revue la plus fréquemment choisie. Il s'agit autant d'articles de jeunes chercheurs et de doctorants que d'auteurs confirmés.

BASE est cependant aussi une revue largement ouverte. Sur les 15 dernières années, bien que près de $60 \%$ des articles publiés soient originaires de Belgique, $26 \%$ proviennent d'Afrique et $8 \%$ du reste de l'Europe.

Quel que soit le sort des articles soumis, le comité de rédaction est particulièrement attentif à apporter des feed-back explicites aux auteurs, leur permettant d'améliorer leur travail de recherche et de rédaction.

\section{QUALITÉ ET TRANSPARENCE}

Avec les évolutions du monde de la publication scientifique évoquées ci-dessus, il semble de plus en plus indispensable de démontrer la qualité du travail scientifique, des processus de validation et d'édition des articles et du fonctionnement de BASE.

Avec l'entrée dans le Directory of Open Access Journals (DOAJ) en 2004, dans le Science Citation
Index en 2008 et dans Scopus en 2009, le travail d'édition s'est fortement professionnalisé, notamment via l'engagement d'une éditrice.

Le logiciel libre OJS (Open Journal System) installé en 2014 permet aux auteurs de compléter eux-mêmes les métadonnées de leurs articles. Lors du dépôt, tous les co-auteurs sont automatiquement informés de la soumission. Au cours du processus, qui comprend une première analyse (scope de la revue, respect du guide des auteurs, aspects éthiques, plagiat et qualité de la langue), plusieurs passages en comité de rédaction et la lecture en double aveugle par des pairs, l'auteur correspondant peut à tout moment suivre l'évolution de son manuscrit.

Outre un guide détaillé des instructions aux auteurs, les sites web de BASE (sur http://www.pressesagro. be et https://popups.uliege.be) présentent avec précision l'ensemble des acteurs avec les spécialités et l'affiliation de tous les membres du comité de rédaction, l'ensemble des points d'attention et les délais fixés pour chaque étape. Une infographie (voir dans le menu « À PROPOS ») aide les utilisateurs de BASE à en avoir une vision d'ensemble.

Enfin, grâce à des suggestions et des demandes explicites de l'Open Access Scholarly Publishers Association (OASPA) et du Committee on Publication Ethics (COPE), le fonctionnement a été sensiblement amélioré en portant une attention particulière à la communication et aux processus de validation et d'édition des articles. En répondant à l'ensemble de leurs critères de qualité, BASE est devenu membre de ces deux organismes en janvier 2018.

\section{BASE ET L'OPEN ACCESS}

Depuis 2004, l'Open Access est aussi un point d'attention pour BASE. Publier en Open Access, ce n'est pas simplement donner accès gratuitement aux articles. C'est, d'une part, rendre accessibles les données de la recherche publique et également, d'autre part, donner aux lecteurs la liberté de copier, distribuer et communiquer les articles et d'adapter, remixer, transformer et créer des contenus à partir de ces articles.

Le DOAJ et l'OASPA privilégient la licence CC BY (CC pour Creative Commons, et BY signifie qu'il faut citer la source utilisée). Cette licence a été adoptée pour les articles publiés dans BASE à partir de 2015. Les limitations introduites par une licence trop fermée (par exemple, pas d'utilisation commerciale ou pas de modification) empêchent inutilement, dans de nombreuses situations, l'utilisation des articles. Utiliser une licence ouverte, comme la licence CC BY, permet une diffusion plus large des connaissances, d'autant que la majorité des recherches publiées dans BASE relèvent de financements publics. 
Le modèle économique de BASE, reposant sur des financements institutionnels et des subventions publiques, permet d'offrir aux lecteurs et aux auteurs un service gratuit. Cet aspect est particulièrement important alors que l'Open Access est trop souvent associé à l'image négative de charges parfois très élevées imputées aux auteurs. Actuellement, les données enregistrées dans le DOAJ, la base de référence en matière d'Open Access, montrent que $30 \%$ des revues font payer les auteurs. BASE, en offrant un service totalement gratuit, veut se démarquer de cette pratique qui ternit l'image de l'Open Access.

\section{BASE ET SES PROCESSUS}

Sur les 331 revues internationales du domaine «Agronomy and Crop Science » reprises par le Scimago Journal \& Country Rank (https://www.scimagojr. com/), BASE est la seule revue en Open Access, avec facteur d'impact, qui publie des articles en anglais et en français. Cette particularité attire régulièrement des articles de moindre qualité (qualité de la langue, bibliographie trop ancienne, pauvreté du dispositif expérimental, sujet trop local, absence d'originalité, etc.) et entraine un taux de rejet moyen sur les cinq dernières années particulièrement élevé, de $83 \%$. En 2017, la durée moyenne de la décision pour le rejet a été de 41 jours. Sur les 223 rejets enregistrés, 33 ont été signifiés aux auteurs dans la semaine et 138 dans le mois. Heureusement, le processus en étapes successives et les réunions mensuelles du comité de rédaction permettent de retirer rapidement du circuit des manuscrits aux déficiences manifestes et d'en informer rapidement les auteurs. Le processus est évidemment plus long pour les manuscrits acceptés. La durée moyenne pour l'acceptation de la version finale d'un manuscrit est de 8 mois.

Comme BASE couvre de nombreux domaines, la liste des reviewers est particulièrement longue (plus de 1000 personnes pour les 10 dernières années). Pour la validation des articles, deux reviewers au minimum sont sollicités. Identifier et obtenir l'accord de deux pairs est un travail de longue haleine. Les scientifiques compétents sont généralement surchargés et trop fréquemment sollicités. Le travail de reviewer est de plus peu reconnu et rarement valorisé dans les mécanismes de promotion des chercheurs et des enseignants.

Il faut en général contacter une dizaine de chercheurs avant d'obtenir les accords nécessaires de relecture. L'auteur correspondant doit fournir une liste de lecteurs potentiels lors de la soumission mais, après analyse, il s'avère bien souvent que ces personnes sont ou ont été en relation avec les auteurs, co-auteurs, membres de comité de thèse ou même collègues.

BASE laisse au reviewer un délai d'un mois. Ce délai est cependant rarement respecté et le comité de rédaction ne dispose, en dehors des rappels, d'aucun moyen pour le raccourcir.

Sur les cinq dernières années, BASE a validé et publié 507 articles dont 81 dans cinq numéros spéciaux. Approximativement, la moitié de ces articles était en français et l'autre en anglais. La proportion des articles en anglais est en légère augmentation (Figure 1).

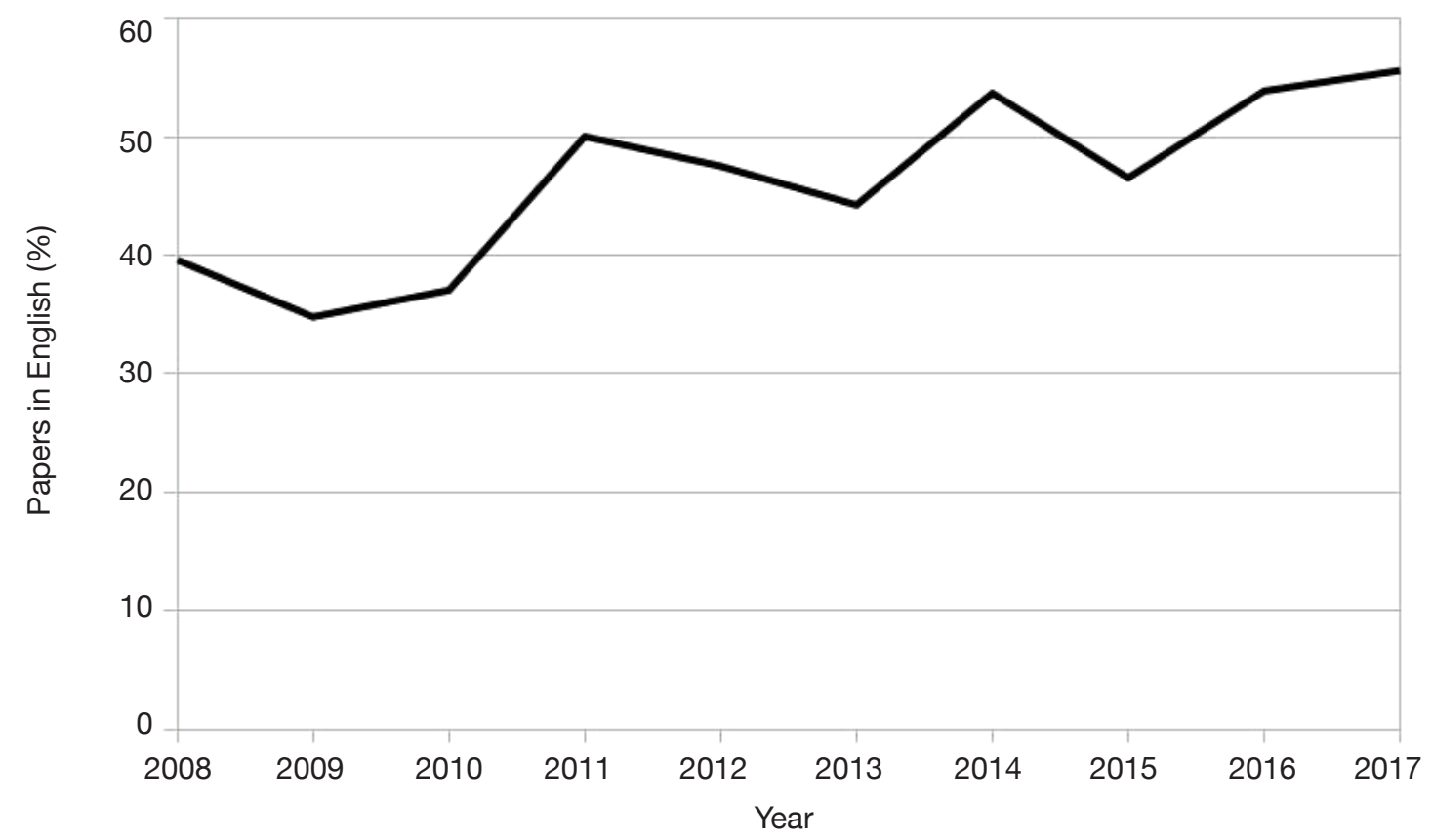

Figure 1. Proportion d'articles en anglais sur les 10 dernières années - Proportion of articles in English over the last 10 years. 


\section{BASE EN QUELQUES CHIFFRES}

Rien que pour l'année 2017, les données OJS indiquent qu'il y a eu 463816 téléchargements d'articles. Pour la même année, les statistiques de PoPuPS, la seconde interface de BASE, indiquent 491592 visualisations d'articles (en $\mathrm{html}$ ). Le nombre total de téléchargements est donc proche du million, sans compter les accès via les plateformes comme EBSCO ou Proquest qui proposent également les textes intégraux. De plus, il est possible d'accéder à certains articles via les dépôts réalisés par les auteurs sur les répertoires institutionnels, comme le site $O R B i$ de l'Université de Liège. La politique d'Open Access est donc une politique qui fonctionne et assure une vaste audience aux chercheurs qui choisissent BASE.

Du côté des bases de données bibliographiques, la revue BASE est reprise dans les principales bases de données bibliographiques généralistes et les bases de données bibliographiques spécialisées. Cette indexation est aussi une importante source de visibilité.

Scopus dénombre 4743 citations sur les 21 volumes publiés. Le nombre de citations est croissant d'année en année (Figure 2). Le dernier facteur d'impact (Clarivate Analytics) publié est l'IF 2017. Il est de 0,79 . Il était de 0,43 l'année dernière. Cette hausse s'explique par le nombre de citations (63) du numéro spécial «AgricultureIsLife ». Les données Scopus et Google Scholar confirment cette croissance des indices de citations. Le CiteScore 2017 de BASE est par exemple passé de 0,62 à 0,93 . Même si ces indices ne constituent pas une fin en soi, ils permettent de cerner la perception de la revue BASE dans le monde scientifique. Paradoxalement, ce facteur d'impact est considéré pour certains domaines comme très modeste, alors que, pour d'autres secteurs scientifiques, il est concurrentiel avec les revues de référence. BASE vit là une de ses ambigüités.

\section{PERSPECTIVES}

Cette description, plutôt positive, ne doit pas faire oublier les difficultés rencontrées, principalement à cause du caractère pluridisciplinaire de BASE. En effet, outre le nombre important de reviewers et la difficulté à faire aboutir rapidement le processus de peer reviewing, l'admission de certains manuscrits doit régulièrement faire l'objet d'un débat. Il est par ailleurs financièrement impossible d'engager un éditeur spécialisé pour chaque domaine. Ce sont les membres du comité de rédaction qui jouent ce rôle.

Même si, de plus en plus souvent, des méga-revues couvrent de larges éventails de domaines de la science, cette multidisciplinarité caractérisant BASE peut s'avérer être un inconvénient car, à la différence des méga-revues, BASE ne publie qu'un nombre limité d'articles par année. Il en résulte que tous les domaines possibles ne sont pas représentés sur un laps de temps restreint, ce qui a pour conséquence que les auteurs, issus ou non de nos institutions, préfèrent souvent soumettre leurs manuscrits à des revues plus spécialisées dont le titre reflète mieux leur domaine de compétence. $\mathrm{Ce}$ choix est aussi guidé par des opportunités de carrière, liées à l'importance que représentent encore le facteur d'impact et le $H$ index dans les processus d'évaluation des scientifiques.

Il n'est cependant pas question de supprimer le côté multidisciplinaire de BASE, c'est son essence même. C'est donc à la qualité du comité de rédaction qu'il

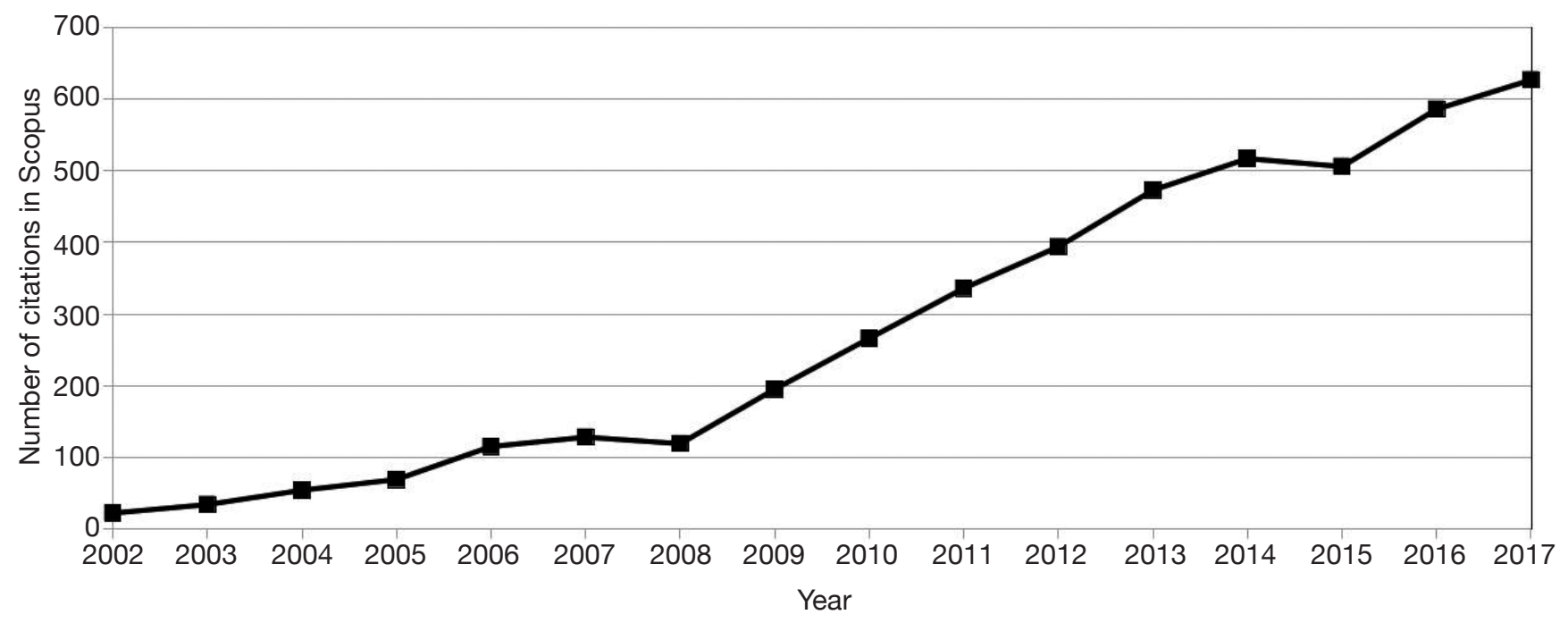

Figure 2. Évolution du nombre de citations dans Scopus depuis 2002 - Evolution of the number of citations in Scopus since 2002. 
faut porter attention. Afin d'en maintenir la dynamique et la représentativité, la liste des membres est revue périodiquement par appel interne. Tous les domaines couverts doivent être maitrisés par au moins un membre.

Enfin, pour une revue dont l'initiative émane d'institutions scientifiques, la communication interne est tout aussi importante que la communication au niveau national et international. Cet article fait partie de cet arsenal de communication. Le soutien des autorités de tutelle en fait également partie.

Afin d'éviter de diluer l'intérêt des lecteurs de BASE, le comité de rédaction va s'attacher à publier des numéros thématiques comme les numéros spéciaux «AgricultureIsLife» ou «2 $2^{\mathrm{e}}$ Atelier Nitrate-Eau» parus récemment. Cela suppose évidemment de recevoir suffisamment de manuscrits de qualité dans un thème donné. Une autre piste consiste à proposer aux jeunes chercheurs la possibilité de soumettre des short notes bien structurées, sur des thèmes innovants.

\section{Bibliographie}

Baudoin J.-P., 1997. Éditorial. Biotechnol. Agron. Soc. Environ., 1(1), 3.

Bon M., 2015. Principles of the self-journal of science: bringing ethics and freedom to scientific publishing. Self J. Sci., 46.

Butler D., 2013. The dark side of publishing. Nature, 495, 433-435.
Callaway E., 2016. Publishing elite turns against impact factor. Senior staff at societies and leading journals want to end inappropriate use of the measure. Nature, $\mathbf{5 3 5}$, 210-211.

Casadevall A. \& Fang F.C., 2014. Causes for the persistence of impact factor mania. MBio, 5(2), 1-6.

Crawford W., 2017. Gold Open Access journals 2011-2016. Livermore, CA, USA: Cites Insights Books.

Galligan F. \& Dyas-Correia S., 2013. Altmetrics: rethinking the way we measure. Ser. Rev., 39(1), 56-61.

Margalida A. \& Colomer M.À., 2016. Improving the peerreview process and editorial quality: key errors escaping the review and editorial process in top scientific journals. PeerJ, 4e 1670.

Murphy F., 2016. An update on peer review and research data. Learn. Publ., 29(1), 51-53.

Pochet B., 2002.Éditorial. Biotechnol.Agron. Soc.Environ., 6(1), 3 .

Pochet B. \& Baudoin J.-P., 2009. Éditorial. Biotechnol. Agron. Soc. Environ., 13(1), 75-76

Satyanarayana K., 2010. Impact factor and other indices to assess science, scientists and scientific journals. Indian J. Physiol.Pharmacol., 54(3), 197-212.

Wenzler J., 2017. Scholarly communication and the dilemma of collective action: why academic journals cost too much. Coll. Res. Libr., February, 183-200.

(13 réf.) 